\title{
Carcinomatosis peritoneal por tumor apendicular
}

\author{
A. Guerrero Zotano, J. Aparicio Urtasun, L. Pellín Ariño, J. Molina Saera, L. Palomar Abad, J. Ponce Lorenzo
}

\section{Resumen}

- Introducción: el término Pseudomixoma Peritoneal es una entidad rara que hace referencia a la diseminación peritoneal de un tumor cuyas células producen gran cantidad de mucina. Suele proceder de un tumor apendicular perforado, y se distribuyen de una forma característica por la superficie peritoneal. Aunque histológicamente puedan no mostrar agresividad, clínicamente sí lo son.

- Material y métodos: presentamos el caso de una mujer diagnosticada de carcinomatosis peritoneal por un carcinoma apendicular. Fue tratada mediante cirugía y quimioterapia sistémica basada en 5FU. De esta forma conseguimos una larga supervivencia con un buen control sintomático.

- Conclusiones: no existen tratamientos de elección para esta enfermedad. Basándose en su peculiar comportamiento clínico, algunos autores intentan una aproximación curativa, mediante cirugías agresivas y quimioterapia intraperitoneal. Se trata de un procedimiento complicado, no validado en ensayos clínicos. El caso que presentamos muestra que una opción razonable de tratamiento puede basarse en cirugía convencional y quimioterapia sistémica.

\section{Palabras clave:}

Pseudomixoma peritoneal. Tumor apendicular. Carcinomatosis peritoneal.

Oncología, 2005; 28 (4):197-201 


\section{Summary}

- Purpose: Mucinous ascites by mucus-producing tumour epithelial cells with peritoneum dissemination is a rare condition known as pseudomyoma peritonaei. In nearly all the cases the cause is a primary tumour of the appendix. It is an entity with multifocal peritoneal epithelial implants, distributed in a characteristic way. Although it can be not aggressive histologically, it is invariably fatal clinically.

- Material and methods: We present the case of a woman diagnosed of peritoneal carcinomatosis by an appendicular carcinoma, who was treated by surgery and systemic chemotherapy based on 5-FU. With this approach we got a long survival with a good symptomatic control.

- Conclusion: There are no standard treatments for this disease. Based on its particular behaviour, the approach of some specialists is an aggressive surgery combined with intraperitoneal chemotherapy. However, it is a complex procedure, not validated by clinical studies. Our case shows that it can be achieved a good clinical control with conservative surgery and systemic chemotherapy.

Key words: Pseudomyxoma peritonaei. Appendiceal tumour. Peritoneal carcinomatosis.

\section{Introducción}

El término Pseudomixoma Peritoneal (PMP) es una entidad clínica caracterizada por la diseminación peritoneal de tumores que característicamente producen mucina. En la inmensa mayoría de los casos el asiento del tumor primario se localiza en un apéndice perforado. Aunque biológicamente poco agresivos, tienen un comportamiento clínico maligno. Producen una cantidad copiosa de ascitis mucinosa, que unido al crecimiento tumoral, suele desembocar en un íleo intestinal. Tradicionalmente el tratamiento tiene un carácter paliativo, basado en cirugías repetidas para aliviar las obstrucciones intestinales drenando la mayor cantidad de moco. Hoy en día hay autores que realizan una aproximación con intención curativa mediante complicadas técnicas quirúrgicas y quimioterapia intraperitoneal. Presentamos a continuación un caso de pseudomixoma peritoneal, con un comportamiento clínico característico, y que fue tratado mediante cirugía y quimioterapia sistémica.

\section{Caso clínico}

Mujer de 56 años, sin antecedentes personales de interés, que consulta en junio de 1995 por dolor y distensión abdominal progresiva de 3 meses de evolución. A la exploración física se constata la existencia de un abdomen distendido, con semiología de ascitis, no a tensión. No hay signos de hepatopatía ni insuficiencia cardíaca. Las exploraciones complementarias muestran una bioquímica básica y hemograma sin alteraciones, unos marcadores tumorales con un Ca 125: $35 \mathrm{Ul} / \mathrm{ml}$, Ca 19.9: $37 \mathrm{Ul} / \mathrm{ml}$ y CEA: $8 \mathrm{Ul} / \mathrm{ml}$. Se intenta realizar paracentesis diagnóstica, pero es infructuosa en varios intentos. Las pruebas de imagen hablan de una TAC abdomino-pélvica que muestra la existencia de ascitis, con adenopatías y varios nódulos peritoneales; una colonoscopia normal y una ecografía ginecológica normal.

Finalmente la paciente se somete a laparotomía exploradora encontrándose una cantidad moderada de ascitis con abundante moco, múltiples adherencias a intestino y anejos ginecológicos, numerosos implantes en omento, pared abdominal y peritoneo $\mathrm{y}$, un apéndice abierto de aspecto mucoso. Se practica un histerectomía con doble anexectomía, linfadenectomía pélvica, apendicectomía y hemicolectomía derecha. La cirugía no es radical, permaneciendo enfermedad residual superior a $2 \mathrm{cms}$. El análisis anatomopatológico de las piezas informa de una carcinomatosis peritoneal por adenocarcinoma de apéndice; colon y ovario sin alteraciones.

La TAC postcirugía muestra nódulos tumorales en 
epiplón y peritoneo. Los marcadores tumorales habían descendido a niveles normales (CEA: 1,7 $\mathrm{Ul} / \mathrm{ml}$; Ca 19.9:27 Ul/ ml).

Se decide administrar quimioterapia con esquema de la Clínica Mayo (5FU-FA). La paciente recibe tratamiento durante un año con un total de 12 ciclos, desapareciendo las imágenes de TAC y con normalización de marcadores. Desde febrero de 1997 permanece en control por el servicio de oncología médica. Durante el seguimiento, se aprecian nódulos peritoneales de lento crecimiento, con un ascenso progresivo de marcadores tumorales. Se opta por la abstención terapéutica, dado que la paciente permanece asintomática. Cuando el crecimiento de los nódulos se hizo manifiesto, se solicitó punción biopsia, en junio de 2001, que confirmó la recaída peritoneal de adenocarcinoma apendicular. En ese momento se decide retratar con esquema Clínica Mayo hasta un total de 8 ciclos, alcanzando criterios de enfermedad estable. Se decide continuar con quimioterapia de tercera línea con Tomudex-Oxaliplatino (6 ciclos). La respuesta, en agosto de 2002, fue nuevamente de estabilización. Desde entonces la paciente permanece oligoasintomática con vida activa, y con la presencia de múltiples nódulos peritoneales que forman una coraza en la pared abdominal (Fig. 1). En diciembre de 2003, la enfermedad progresa produciéndose compresión de cola de caballo secundaria a masa presacra. Recibe radioterapia paliativa, aliviándose el dolor y sin producirse déficit neurológico. La situación de enfermedad a octubre de 2004,

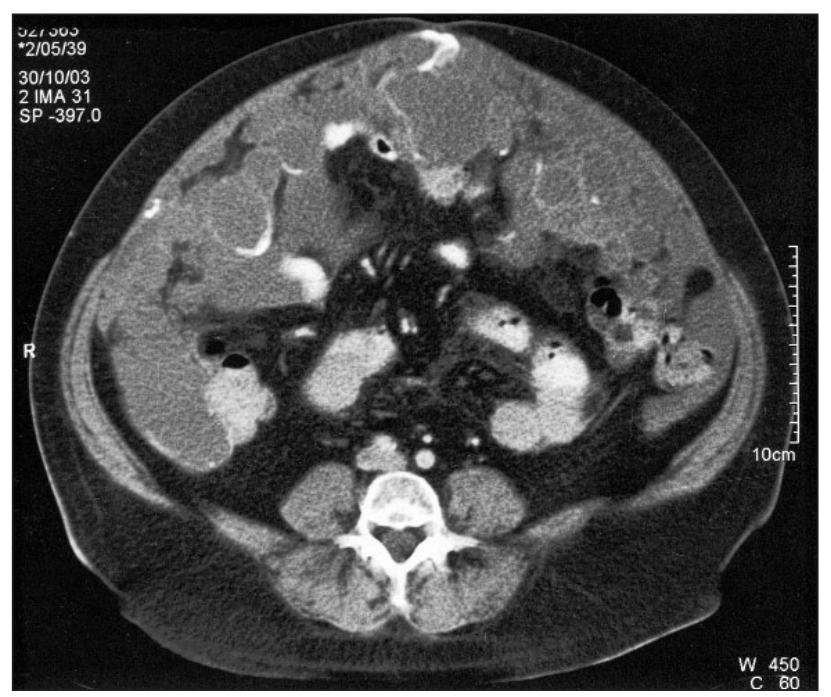

Figura 1. TAC abdominal ilustrativa de carcinomatosis peritoneal. es la de lento deterioro del estado general por progresión de enfermedad, con anemia y desnutrición por dificultad en la ingesta debido a cuadros suboclusivos.

\section{Discusión}

La presencia de ascitis gelatinosa debido a la diseminación peritoneal de células epiteliales productoras de mucina recibe el nombre de pseudomixoma peritoneal $(\mathrm{PMP})^{1}$. En prácticamente la totalidad de los casos, el origen de estas células son tumores de apéndice. Estos tumores apendiculares son de potencial maligno variable, desde adenomas a adenocarcinomas, y tienen un comportamiento distinto al resto de neoplasias gastrointestinales. Biológicamente son poco agresivos, tienden a recubrir la superficie peritoneal más que a penetrar en los tejidos, no suelen metastatizar ni producir afectación ganglionar. Diseminan al peritoneo en estadios tempranos de la enfermedad, debido a una ruptura en la pared del apéndice. La siembra peritoneal se distribuye de forma característica en lugares donde se reabsorbe el líquido ascítico (cara abdominal del hemidiafragma derecho, omentos mayor y menor, espacios retrohepático e infrahepático derechos y pelvis), respetando las superficies peritoneales con mayor actividad peristáltica, como el intestino delgado y en menor medida el estómago.

Aunque la histología sea de bajo potencial maligno, son neoplasias incurables e inevitablemente fatales sin tratamiento. Su historia natural se caracteriza por un crecimiento lento y progresivo que produce compresión de las estructuras abdominales, falleciendo la mayor parte de los enfermos por obstrucción intestinal.

Históricamente ha existido cierta confusión para clasificar esta entidad, debido a discordancias entre la histología del tumor primario y el potencial maligno del cuadro clínico. Ronnet arroja luz sobre el asunto, mediante una clasificación basada en las características histológicas de los implantes peritoneales y la naturaleza del tumor primario, que posteriores trabajos han confirmado como de utilidad pronóstica ${ }^{2,3}$. Podemos clasificar un cuadro de PMP en alguno de los siguientes grupos histopatológicos:

1. Adenomucinosis: implantes peritoneales con mínima atipia y actividad mitótica, pero capaces de 
producir abundantes cantidades de ascitis mucinosa. Son tumores superficiales, no invasivos. El tumor primario suele corresponder a un adenoma mucinoso apendicular.

2. Carcinomatosis mucinosa peritoneal: incluye implantes peritoneales con abundante mucina pero cuyo epitelio tiene características citológicas de carcinoma, con células en anillo de sello. Produce invasión de órganos vecinos, linfadenopatías y pueden metastizar más allá de la cavidad abdominal. El tumor primario suele corresponder a un adenocarcinoma apendicular u otra neoplasia gastrointestinal.

3. Tipo intermedio: entidad con predominio de adenomucinosis pero con áreas focales de adenocarcinoma.

El análisis de diversas series de pacientes tratados con cirugía ha permitido identificar la calidad de la cirugía como otro factor pronóstico de primer orden, independiente de la histología. Con una citorreducción óptima, entendida como tal la ausencia de tumor o la presencia de implantes menores de $2.5 \mathrm{~cm}$, independientemente del número y su localización, la mediana de supervivencia a 5 años está en torno al $80 \%$, mientras que si la citorreducción no es adecuada, ésta no supera el $20 \%$.

Tradicionalmente, el tratamiento de esta enfermedad ha sido la cirugía, mediante repetidas intervenciones ante las eventuales recidivas que buscaban drenar la ascitis gelatinosa y eliminar la mayor cantidad de tumor. Se consiguen de esta forma intervalos libres de enfermedad en torno a 2.5 años, y una supervivencia global a 5 años de $3 \%$ al 50\%, según las series, sin curaciones a largo plazo, independientemente del subtipo histológico ${ }^{4,5}$.

Basados en los hechos de que son neoplasias no invasivas, que quedan limitadas a la cavidad abdominal, y que se distribuyen de una forma característica que permite resecciones completas sin comorbilidad excesiva (el intestino delgado raramente está afecto), ha llevado a muchos autores a considerar que el objetivo de este tratamiento puede ser curativo, y no paliativo como antaño ${ }^{6}$. Existen, desde mediados de los años 80, diversos estudios con amplias series de pacientes en los que se aplican procedimientos agresivos basados en cirugías citorreductoras que incluyen peritonectomías y quimioterapia hipertérmica intraperitoneal (mitomicina-c y 5FU) para eliminar cualquier evidencia de enfermedad que pudiese quedar en la cavidad abdominal tras la ciru- gía. La serie más grande publicada en la literatura es la de Sugarbaker, que ha tratado 385 pacientes mediante este complicado procedimiento, incrementando considerablemente las tasas de supervivencia ${ }^{7}$. Así, pacientes con una citorreducción completa e histología de adenomucinosis alcanzan supervivencias a 5 años del $86 \%$, y en torno al $50 \%$ si la histología es adenocarcinoma o mixta. De esta forma se consiguen curaciones, y disminuye la necesidad de laparotomías sucesivas. Aún con todo, hay que tener en cuenta que las complicaciones del procedimiento no son desdeñables, con tasas de morbilidad grados IIl y IV de un $27 \%$, siendo lo más frecuentes pancreatitis (7\%) y fistulización (4\%).

La complejidad de la quimioterapia intraperitoneal hipertérmica y la toxicidad encontrada hacen difícil la generalización de este tratamiento, fuera de centros especializados con experiencia, sobre todo teniendo en cuenta que va a ser complicado la realización de estudios comparativos que confirmen esa ventaja en la supervivencia. Existen pocos datos sobre el uso de quimioterapia sistémica en esta enfermedad $^{8,9}$ y su utilidad es cuestionada sobre todo en histologías no invasivas, dada la dificultad en alcanzar la superficie peritoneal desde la circulación general. El tipo de quimioterapia más empleada esta basada en 5FU, pero su eficacia es difícil de determinar dada la escasez de datos en la literatura.

El caso que presentamos viene a mostrarnos varios aspectos que hamos comentado sobre este tipo de neoplasias. Por un lado, que se trata de un tumor menos agresivo que el resto de neoplasias gastrointestinales; así, nuestra paciente, a pesar de haber debutado con una carcinomatosis peritoneal, tiene una supervivencia de casi 10 años, algo impensable si se tratase de un cáncer gástrico o pancreático con siembra peritoneal. En cuanto al tratamiento de la enfermedad, por lo que conocemos de este tumor, el esfuerzo quirúrgico es capital, algo parecido a lo que ocurre en los tumores de ovario; pero existen ciertos recelos a la hora de adoptar como tratamiento estándar la quimioterapia intraperitoneal perioperatoria, como abogan reconocidos expertos en la materia. En el carcinoma de ovario, tumor de comportamiento comparable, aún no ha podido demostrarse la superioridad de Ia quimioterapia intraperitoneal frente a la sistémica ${ }^{10,11}$. Por otra parte la supervivencia alcanzada en la experiencia de Sugarbaker con el uso de quimioterapia intraperitoneal, comparada con 
otras series, parece especialmente ventajosa en la histología de mejor pronóstico (adenomucinosis), mientras que es sólo ligeramente superior cuando hablamos de histologías de peor pronóstico. El caso que presentamos, al igual que otros existentes en la literatura, viene a mostrar que es posible alcanzar largas supervivencias, a pesar de histología agresiva, tras una cirugía citorreductora y con el uso de quimioterapia sistémica basadas en 5FU.

Correspondencia:

Dr. J. Aparicio Urtasun

Servicio de Oncología Médica Hospital Universitario $\mathrm{La} \mathrm{Fe}$

Avda. Campanar, 21

E-46009 Valencia

E-mail: aparicio_josurt@gva.es

\section{Bibliografía}

1. Sugarbaker PH, Ronnett BM, Archer A, et al. Pseudomyxoma peritonei syndrome. Adv Surg 1997; 30:233-80.

2. Ronnett BM, Zahn CM, Kurman RJ, et al. Disseminated peritoneal adenomucinosis and peritoneal mucinous carcinomatosis. A clinicopathologic analysis of 109 cases with emphasis on distinguishing pathologic features, site of origin, prognosis, and relationship to "pseudomyxoma peritonei”. Am J Surg Pathol 1995; 19:1390-408.

3. Jackson SL, Fleming RA, Loggie BW, et al. Gelatinous ascites: A cytohistologic study of pseudomyxoma peritonei in 67 patients. Mod Pathol 2001; 14:664-71.

4. Gough DB, Donohue JH, Schutt AJ, et al. Pseudomyxoma peritonei: longterm patient survival with an aggressive regional approach. Ann Surg 1994; 219:112-9.

5. Nitecki SS, Wolff BG, Schlinkert R, Sarr MG. The natural history of surgically treated primary adenocarcinoma of the appendix. Ann Surg 1994; 219:51-7.

6. Sugarbaker PH. Observations concerning cancer spread within the peritoneal cavity and concepts supporting an ordered pathophysiology. Cancer Treat Res 1995; 82:79-99.

7. Sugarbaker PH, D Chang. Results of treatment of 385 patients with peritoneal surface spread of appendiceal malignancy. Ann Surg Oncol 1999 6(8):727-31.

8. Ronnet BM, Yan H, Kurman RJ, et al. Patients with pseudomyxoma peritonei associated with disseminated peritoneal adenomucinosis have a significantly more favourable prognosis than patients with peritoneal mucinous carcinomatosis. Cancer 2001; 92:85-91.

9. Chang D, Levitz JS, Sugarbaker PH. Pseudomyxoma peritonei: response to capecitabine. J Clin Oncol 2004; 22:1518-23.

10. Alberts DS, Liu PY, Hanningan EV, et al. Phase III study of intraperitoneal cisplatin (CDDP)/intravenous (IV) cyciophosphamide(CPA) vs IV CDDP/IV CPA in patients with optimal disease stage III ovarian cancer: a SWOGGOG-ECOG intergroup study. Proc Am Soc Clin Oncol 1995; $14: 273$

11. Kirmani S, Braly PS, McClay EF, et al. A comparison of intravenous versus intraperitoneal chemotherapy for the initial treatment of ovarian cancer. Gynecol Oncol 1994; $54: 338-44$ 